\title{
HRTEM and XPS Characterization of Photocatalytic Fe Doped Tioz Nanocrystals Enabling Significant Improvement in the Cleaning of Wastewater
}

\author{
Vahid Moradi, Arthur Blackburn and Rodney Herring \\ CAMTEC, MENG, University of Victoria, Victoria, Canada
}

The information obtained from High Resolution Transmission Electron Microscopy (HRTEM) and analytical X-ray Photo-electron Spectroscopy (XPS) of metal doped titanium dioxide $\left(\mathrm{M}-\mathrm{TiO}_{2}\right)$ nanocrystals targeted as a photocatalyst enabled a significant improvement in their photocatalytic activity for cleaning wastewater. Here, iron (Fe) was used as the metal dopant, as $\mathrm{Fe}$ doped $\mathrm{TiO}_{2}$ should theoretically have a very high photocatalytic efficiency using visible light, due to iron reducing the bandgap of $\mathrm{TiO}_{2}$ from $\sim 3.2 \mathrm{eV}$ to $\sim 2.7 \mathrm{eV}$. Furthermore, high efficiency should result from the most stable oxidation state of iron, +3 , being readily able to accept holes or electrons that can percolate to the nanocrystal surface to aid the production of radicals in the surrounding medium. However, prior to this work, this has not been experimentally the case. Resolving and understanding this problem, as we have done here thus paves the way towards reducing our environmental impact on rivers, lakes and oceans, and so should eventually help in preventing red tides and reducing the acidification of our waters.

Imaging freshly made $\mathrm{Fe}$ doped $\mathrm{TiO}_{2}$ nanoparticles revealed the existence of a thin amorphous contamination layer on their surfaces (Figure 1a). Cleaning the surface of the Fe doped $\mathrm{TiO}_{2}$ nanoparticles using a $\mathrm{HCl}$ acid etch removed a surface contamination layer (Figure 1b). This resulted in the atomic planes of the Fe doped $\mathrm{TiO}_{2}$ nanocrystals extending to the surface, thus improving charge transport to the particle - wastewater interface and thus the chemical activity and efficiency of the cleaning process. As detection of the nature of this exceptionally thin, few atomic layer 'contamination', could not be conclusively revealed by electron energy loss spectroscopy in the TEM, highly surface sensitive XPS was used to gain information on the surface layer. This indicated that the contamination layer was iron oxide (Figure 2) remaining from the doping of the $\mathrm{TiO}_{2}$ by ferric nitrite $\left(\mathrm{Fe}\left(\mathrm{NO}_{3}\right)_{3} .9 \mathrm{H}_{2} \mathrm{O}\right)$ in the sol gel nanoparticle formation process. Knowing that cleanliness of the surface was essential, a double acid etch washing of the crystals was performed resulting in cleaning most of the pollutants from water (Figure 3) [1].

This surface layer identification and removal, which thus gives clean crystalline surface facets to the Fe doped $\mathrm{TiO}_{2}$ particles, now enables visible light rather than UV, which is currently employed in many water cleaning facilities, to be used in photocatalytic water cleaning processes. This should greatly reduce the cost of cleaning water, by using $\sim 97 \%$ efficient LEDs as opposed to the low efficiency UV sources currently used as the excitation source. Further, the general application of Fe doped $\mathrm{TiO}_{2}$ nanocrystals for cleaning of water is expected to be improved, as now low cost visible light transmissive cost glasses can be used in the supporting apparatus, as opposed the higher cost UV transmissive materials currently being used.

References:

[1] V. Moradi, M.B.G. Jun, A. Blackburn, R.A. Herring, Applied Surface Science 427 (2018), p. 791. 

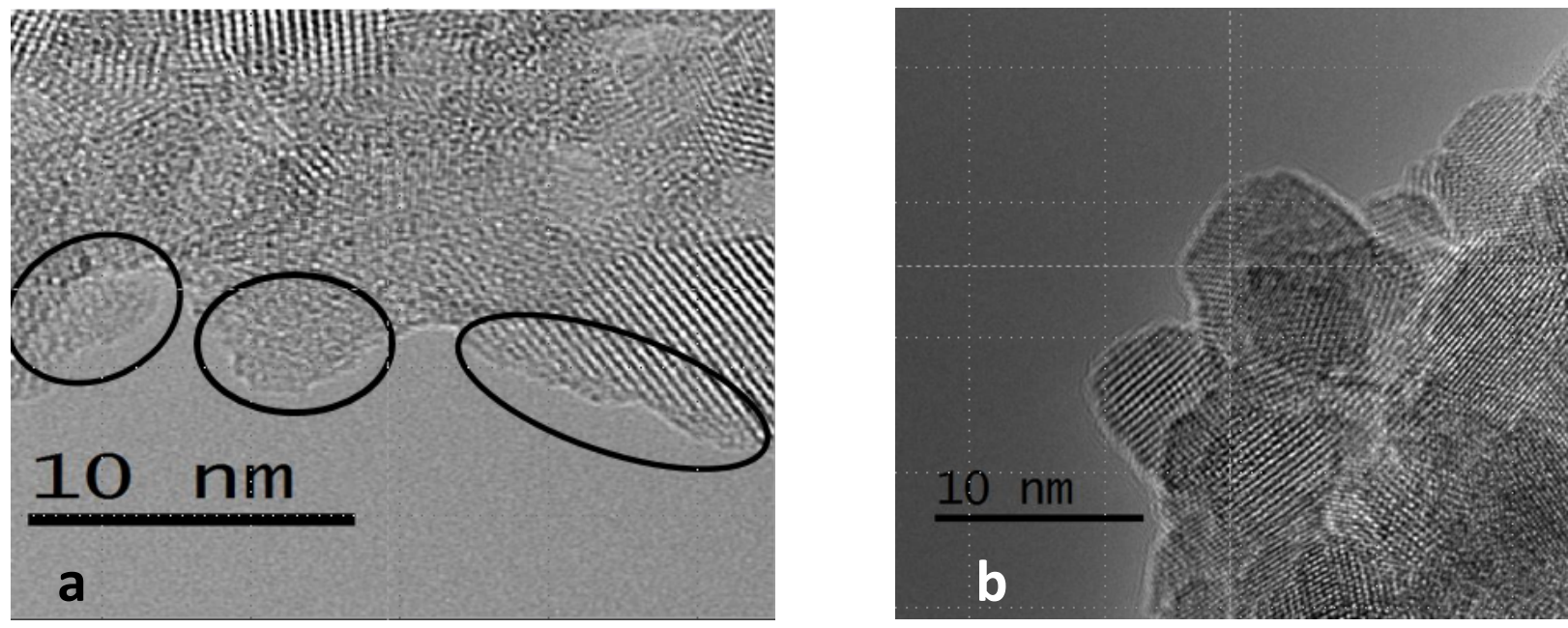

Figure 1. a) HRTEM revealing the existence of a thin amorphous contamination layer on the surface of Fe doped $\mathrm{TiO}_{2}$ particles within the elliptically encircled areas (Figure 1a), b) after $\mathrm{HCl}$ acid cleaning of the amorphous layer, the atomic planes of the $\mathrm{Fe}$ doped $\mathrm{TiO}_{2}$ particles extend to the surface of the $\mathrm{Fe}$ doped $\mathrm{TiO}_{2}$ crystals.

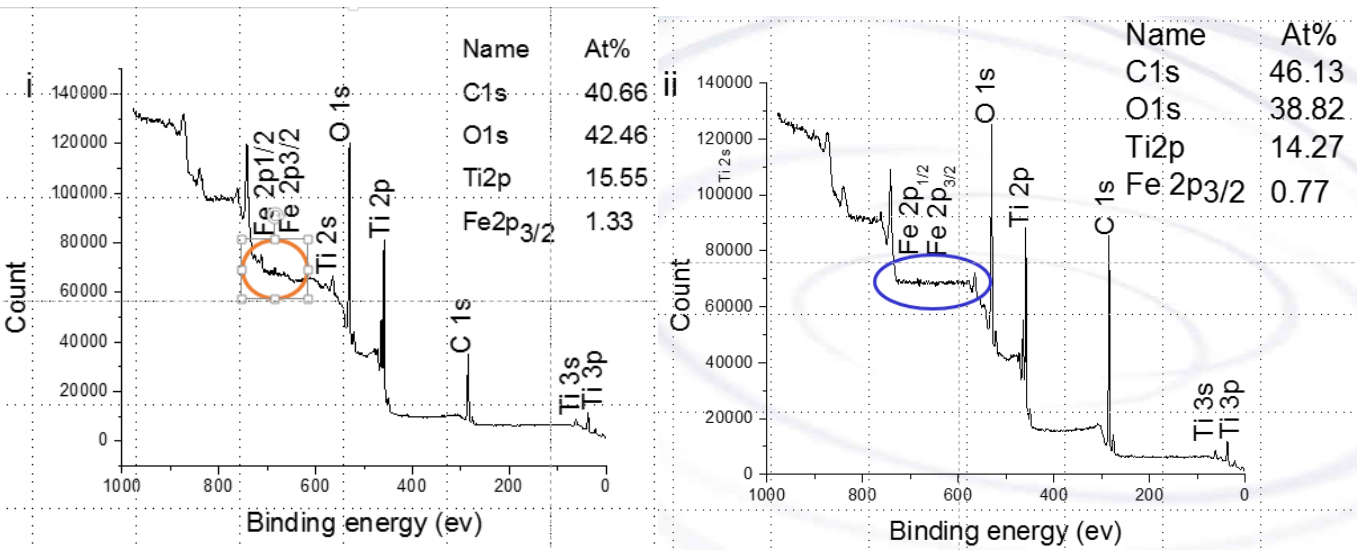

Figure 2. XPS having within the circled areas a) the uncleaned $\mathrm{Fe}$ doped $\mathrm{TiO}_{2}$ showing the presence of $\mathrm{FeO}$ and in $\mathrm{b}$ ) the acid cleaned $\mathrm{Fe}$ doped $\mathrm{TiO}_{2}$ showing the absence of $\mathrm{FeO}$.

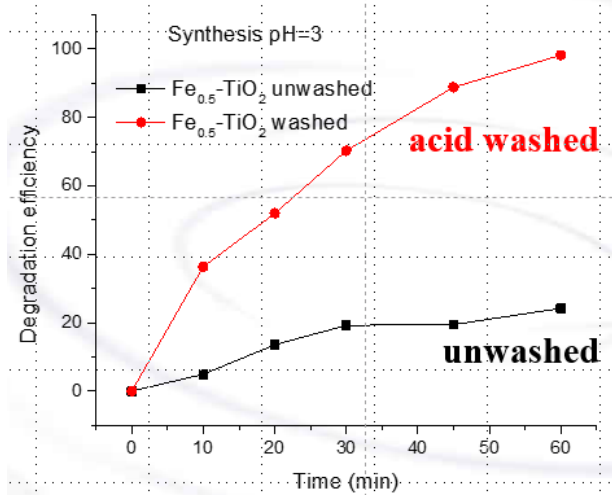

Figure 3. Enhanced photocatalytic degradation efficiency of the acid cleaned $\mathrm{Fe}$ doped $\mathrm{TiO}_{2}$ nanoparticles resulting from HREM imaging that found a contamination layer of the surface of the uncleaned particles. 